\section{Pacific Northwest}

National Laboratory

Operated by Battelle for the

U.S. Department of Energy

\title{
Combining Multiple-Module Output Boundary Conditions to Produce a Single- Input-Module Boundary Condition in FRAMES
}

\author{
G. Whelan \\ K. J. Castleton \\ J. W. Buck \\ R. Y. Taira \\ G. M. Gelston \\ D. L. Strenge
}

October 2006

\author{
Prepared for \\ Engineer Research and Development Center \\ U.S. Army Corps of Engineers \\ Vicksburg, MS \\ under Contract DE-AC05-76RL01830
}




\title{
DISCLAIMER
}

This report was prepared as an account of work sponsored by an agency of the United States Government. Neither the United States Government nor any agency thereof, nor Battelle Memorial Institute, nor any of their employees, makes any warranty, express or implied, or assumes any legal liability or responsibility for the accuracy, completeness, or usefulness of any information, apparatus, product, or process disclosed, or represents that its use would not infringe privately owned rights. Reference herein to any specific commercial product, process, or service by trade name, trademark, manufacturer, or otherwise does not necessarily constitute or imply its endorsement, recommendation, or favoring by the United States Government or any agency thereof, or Battelle Memorial Institute. The views and opinions of authors expressed herein do not necessarily state or reflect those of the United States Government or any agency thereof.

\author{
PACIFIC NORTHWEST NATIONAL LABORATORY \\ operated by \\ BATTELLE \\ for the \\ UNITED STATES DEPARTMENT OF ENERGY \\ under Contract DE-AC05-76RL01830
}

Printed in the United States of America

Available to DOE and DOE contractors from the

Office of Scientific and Technical Information

P.O. Box 62, Oak Ridge, TN 37831-0062;

ph: (865) 576-8401

fax: (865) 576-5728

email: reports@adonis.osti.gov

Available to the public from the National Technical Information Service, U.S. Department of Commerce, 5285 Port Royal Rd., Springfield, VA 22161 ph: (800) 553-6847

fax: (703) 605-6900

email: orders@ntis.fedworld.gov

online ordering: http//www.ntis.gov/ordering.htm 
PNNL-16130

\section{Combining Multiple-Module Output Boundary Conditions to Produce a Single-Input-Module Boundary Condition in FRAMES}
G. Whelan
K. J. Castleton
J. W. Buck
R. Y. Taira
G. M. Gelston
D. L. Strenge

October 2006

Prepared for

Engineer Research and Development Center

U.S. Army Corps of Engineers

Vicksburg, MS

under Contract DE-AC05-76RL01830

Pacific Northwest National Laboratory

Richland, Washington 99352 


\section{Summary}

The Framework for Risk Assessments in Multimedia Environmental Systems (FRAMES), developed at Pacific Northwest National Laboratory, is a Windows ${ }^{\mathrm{TM}}$-based software platform that provides an interactive user interface and, more importantly, specifications to allow a variety of DOS- and Windows-based codes to be integrated within a single framework. The major components of FRAMES include modules (module user interface, analysis code, and potentially pre- and/or post-processors), the Framework User Interface (FUI), sensitivity/uncertainty module, and data-visualization tools. Modules can accept data from the user or other modules and can calculate some portion of the risk assessment. The FUI allows the user to interact with the system. The sensitivity/uncertainty module allows the user to conduct a Monte Carlo analysis, and the visualization tools allow the user to review results from a particular stage in the process.

Many models only expect and consume one set of input from an upstream model. There are instances though when a downstream model is required to consume input from several upstream models. A need was identified to increase the functionality of FRAMES by developing the capability to combine the outputs of multiple modules. A new component, called the Plus Operator, has been created to meet this need. Typically, many modules are needed to cover all the steps required in the risk-assessment process. For example, for a multimedia environmental assessment, the required modules include the type of contaminant selection, the source release, fate and transport (groundwater, vadose zone, surface water, air, overland), the exposure pathway (farm food chain, ingestion, inhalation, dermal, external), and risk (dose, cancer incidence or fatalities, and hazard quotient). The Plus Operator provides the capability to create realistic scenarios for assessments that require combining contaminant 1) fluxes from different sources into one environmental medium or 2) concentrations from different exposure routes at a single receptor.

The Plus Operator thus provides a mechanism to group modules of similar output so that the output can be combined and supplied to downstream modules. This document provides requirements, the design, data-file specifications, the test plan, and the quality assurance/quality control (QA/QC) protocol for the Plus Operator. The requirements identify the attributes of the software. The design describes how the software will be structured to meet those requirements. The specification presents the specific modifications to FRAMES to meet the requirements and design. The test plan confirms that the basic functionality listed in the requirements (black-box testing) actually functions as designed, and QA/QC confirms that the software meets the client's needs. 


\section{Acknowledgments}

The authors would like to extend their appreciation to Dr. Mark Dortch and Mr. Jeff Gerald of the U.S. Army Corps of Engineers, Engineer Research and Development Center (ERDC) in Vicksburg, MS, for supporting this work. Thanks are also extended to Dr. James G. Droppo, Jr. for reviewing the material and Mr. Wayne Cosby for the final edit of the document. This work was funded by ERDC under Contract DE-AC05-76RL01830. 


\section{Acronyms and Abbreviations}

AFF

ATO

DOS

EPA

EPF

ERDC

FRAMES

FUI

GID

ICRP

NCRP

OCRWM

PNNL

QA/QC

RIF

WCF

WFF
Air Flux File, containing time-varying contaminant emissions to the air

Atmospheric Transport Output file, containing chronic or acute time- and spatially-varying concentrations, deposition rates, and external dose (for radionuclides)

Disk Operation System, basic operation system on the computer

U.S. Environmental Protection Agency

Exposure Pathway File, containing concentrations for each exposure pathway

Engineer Research and Development Center

Framework for Risk Analysis in Multimedia Environmental Systems

Framework User Interface

Global Input Data File, contains all of the input data to run modules

International Commission on Radiological Protection

National Council on Radiological Protection and Measurements

Office of Civilian Radioactive Waste Management

Pacific Northwest National Laboratory

Quality Assurance and Quality Control, processes that confirm the quality of the product

Receptor Intake File, containing dose rates for each exposure pathway

Water Concentration File, containing time-varying contaminant concentrations

Water Flux File, containing time-varying water and contaminant fluxes 


\section{Contents}

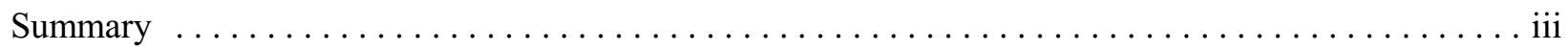

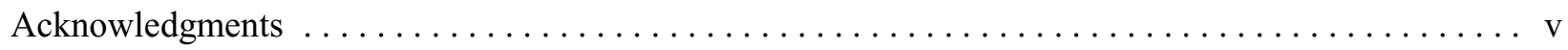

Acronyms and Abbreviations $\ldots \ldots \ldots \ldots \ldots \ldots \ldots \ldots \ldots \ldots \ldots \ldots \ldots \ldots \ldots \ldots \ldots \ldots \ldots \ldots \ldots$

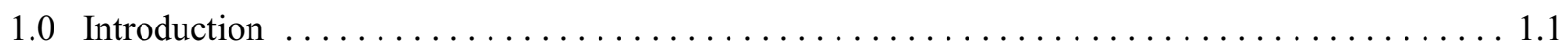

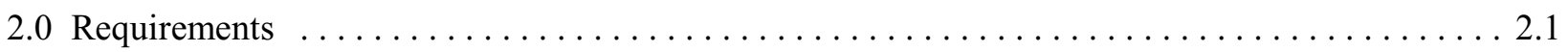

2.1 Framework User Interface Requirements for Inclusion of the Plus Operator $\ldots \ldots \ldots \ldots \ldots 2.1$

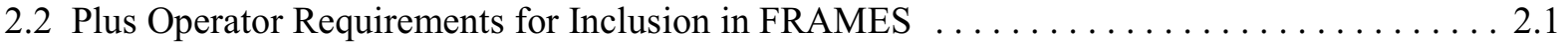

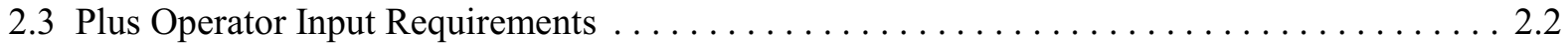

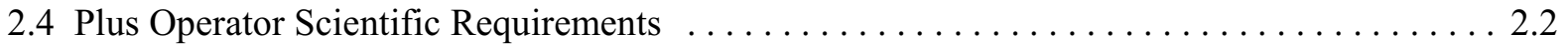

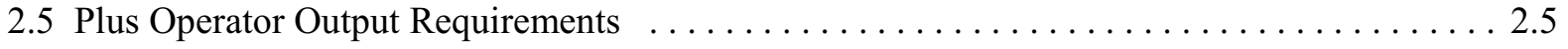

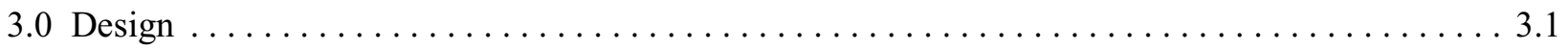

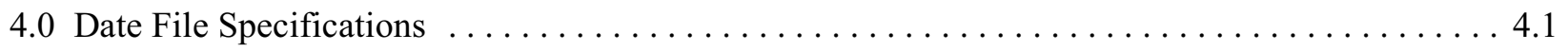

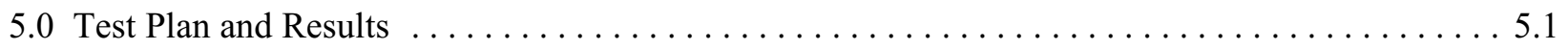

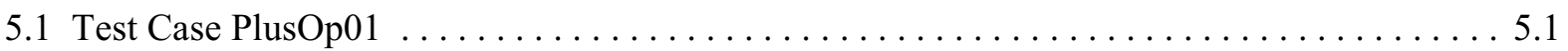

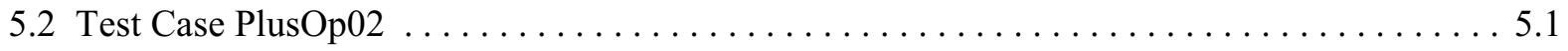

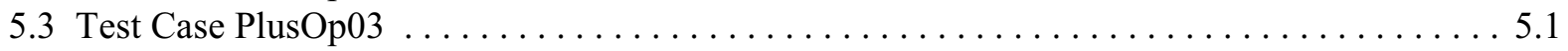

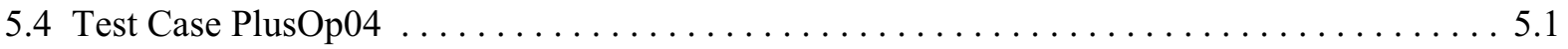

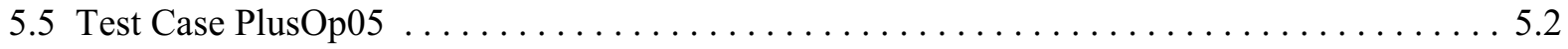

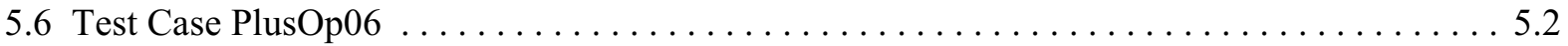

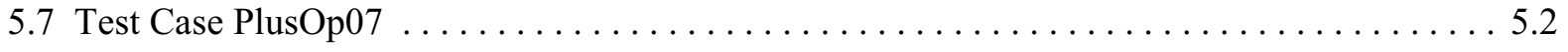

6.0 Quality Assurance Program $\ldots \ldots \ldots \ldots \ldots \ldots \ldots \ldots \ldots \ldots \ldots \ldots \ldots \ldots \ldots \ldots \ldots \ldots \ldots \ldots \ldots \ldots \ldots \ldots .1$

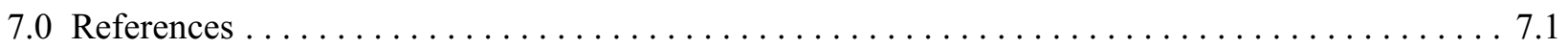




\section{Figures}

1.1. Application of the Plus Operator to Two Aquifer Plumes Contaminating the Same Well

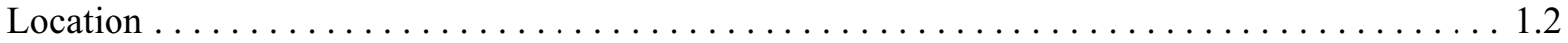

2.1. Approach to Account for Overlapping Areas when Combining like Overland or Surface-Water

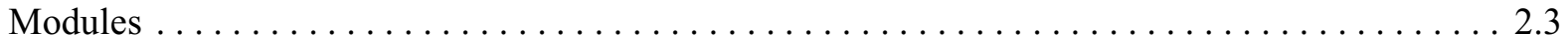

3.1. Conceptual Model Developed by User in FRAMES $\ldots \ldots \ldots \ldots \ldots \ldots \ldots \ldots \ldots \ldots \ldots .1$

3.2. Plus Operator Combining WCF Results from Two Different Aquifer Modules to a Receiving

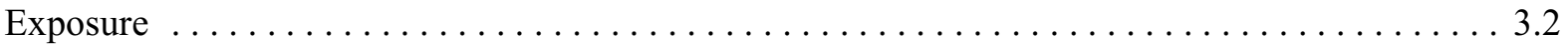

3.3. Conceptual Model With Plus Operator Added by the FUI, Based on the User's Original

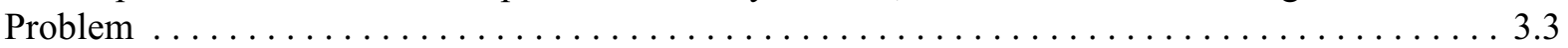

3.4. Virtual Modules Created by the FUI to Implement the Plus Operator for the Existing

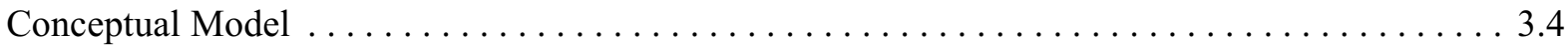

6.1. Confirming Quality in the Environmental Software Development Process . . . . . . . . . 6.2

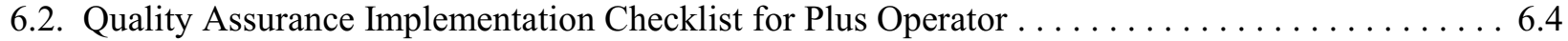

\section{Tables}

6.1. Relationship of PNNL Environmental Software Development Process to Quality Assurance Requirements 


\subsection{Introduction}

The Framework for Risk Assessments in Multimedia Environmental Systems (FRAMES), developed at Pacific Northwest National Laboratory, provides the capability to integrate a variety of DOS and Windows-based environmental codes. FRAMES as a Windows ${ }^{\mathrm{TM}}$-based software platform includes the Framework User Interface (FUI), software modules, the sensitivity/uncertainty module, and data viewers. The FUI allows the user to interact with the system. Software modules include module-specific user-interfaces, environmental model codes, and various pre- and post-processors. The sensitivity/uncertainty module provides the capability to conduct Monte Carlo analyses. The data viewers provide the capability of visual inspection of inputs as well as intermediate and final results. This use of an interactive user interface with underlying linkage specifications for connecting media in a single framework makes FRAMES a powerful tool for conducting environmental risk assessments.

FRAMES is designed to combine the outputs from multiple modules, and a need was identified to facilitate this process. A new component, called the Plus Operator, has been created to meet this need. When a downstream module is designed to only allow a single input data stream, yet multiple events are contributing to the input data stream, the Plus Operator provides a mechanism to capture these multiple boundary conditions without violating the legacy model's input requirements. For example, the Plus Operator would allow an individual to be exposed to contaminated water from two separate water sources if the exposure module was designed to only accept one input data stream. The Plus Operator provides the capability to create realistic scenarios for assessments that require combining contaminant 1) fluxes from different sources into one environmental medium or 2) concentrations from different exposure routes at a single receptor. Therefore, consistency must be maintained between the contaminant flux rate (Qc), the transporting medium (e.g., water or air) flux rate (Q), and contaminant concentration (C), as the direct relationship between these three parameters (i.e., $\mathrm{Qc}=\mathrm{Q} \times \mathrm{C}$ ) provides an important constraint for the merging process. These three parameters cannot be individually superimposed and still maintain their multiplicative relationship. The situation will dictate which parameters can be superimposed and which need to be computed from the superimposed parameters. These combinations include the following:

1. Contaminant fluxes (e.g., in $\mathrm{g} / \mathrm{yr}$ ) from different sources into one environmental medium. When fluxes are superimposed, the control volume is not necessarily considered rigid. This assumption is traditionally used for combining overland flows and surface water flows. Only the same chemicals at the same times can be superimposed.

2. Environmental-medium concentrations of contaminants (e.g., in $\mathrm{mg} / \mathrm{L}$ or $\mathrm{mg} / \mathrm{m}^{3}$ ) at the same medium location. Concentrations in environmental media at the same location are traditionally combined using superposition because the control volume is considered the same for multiple concentrations. Only the same chemicals at the same times can be superimposed.

3. Contaminant concentrations in the same "foodstuff"(e.g., in $\mathrm{mg} /[\mathrm{kg}$ foodstuff solids], $\mathrm{mg} /[\mathrm{L}$ liquid], or $\mathrm{mg} /\left[\mathrm{m}^{3}\right.$ air $]$ ) associated with the same individual or population. Consumption of various foodstuffs via different exposure routes containing contaminant concentrations does not lend itself to superposition in determining exposure. Successful superposition requires the same foodstuff medium (e.g., tomatoes, water, air, or soil) and the same concentration units. Only the same chemicals at the same times can be superimposed.

4. Dose (e.g., in $\mathrm{mg} / \mathrm{kg} / \mathrm{d}$ ) to specific individuals. Dose to specific individuals through various exposure pathways (i.e., inhalation, ingestion, dermal contact, and external dose) represents a rate that could lend itself to superposition, assuming the same person or population, the same exposure pathway (i.e., 
inhalation, ingestion, dermal contact, or external dose), the same foodstuff type (e.g., tomatoes, milk, meat, water, soil), the same chemical, the same times, and the same dose-rate units. Different foodstuff media cannot be combined (e.g., tomatoes and water).

5. Risk to individuals from different exposure routes and pathways. Like risks from different routes of exposure are traditionally combined with superposition. Superposition is by chemical risk, radionuclide risk, or chemical hazard. Superposition is across chemicals, but at the same times, within each risk/hazard category. Chemicals and radionuclides cannot be combined because two fundamentally different approaches (e.g., those of the U.S. Environmental Protection Agency and those of the NCRP/ICRP $\left.{ }^{(a)}\right)$ are sometimes used to compute risk.

In a linear system, combining like outputs involves superposition. Currently, when the multiple module icons within FRAMES are linked to a single downstream module icon, the information is kept separate, and separate calculations are performed. The principles of superpositioning have been used to develop a Plus Operator, which is represented by a system icon that combines like-files and produces a single set of outputs for the multiple sets of inputs it consumes.

Figure 1.1 illustrates the application of the Plus Operator to two aquifer (AQU) plumes contaminating the same well location. To address the concerns associated with combining like outputs from similar models, the design of five separate Plus Operator "modules" is presented. These Plus Operator modules were envisioned to operate as follows:

1. Water Flux Files (WFFs): Two situations exist when combining WWFs. In each instant, water flow rates and contaminant mass flux rates MUST be supplied, even if one is not used. Superposition is viable for the same chemical and the same times for the following.

a. Superposition of water flow rates and mass flux rates. This approach is primarily used to combine output from overland modules or surface water modules.

b. Superposition of water flow rates and concentrations. In this situation, the mass flux rates are computed from water flow rates and concentration. This approach is primarily used for combining the outputs from vadose zone modules or aquifer modules.

2. Water Concentration Files (WCFs): Superposition on contaminant concentrations in water. Superposition is viable for the same chemical and same times.

3. Air Transport Output (ATO) files: Superposition on deposition rate and air concentration (note that the model would be charged with taking the deposition rate and multiplying it times the deposition area to obtain the mass flux rate to the soil). Superposition is viable for the same chemical and the same time for the models that produce concentrations associated with chronic exposures.

4. Exposure Pathway Files (EPFs): A limited set of superposition cases is possible for EPFs. Superposition is viable for the same foodstuff medium (e.g., tomatoes, water, air, or soil), the same chemical, the same times, the same location, and the same concentration units. When referring to the same foodstuff medium, superposition is implemented on the exact same foodstuff (e.g., exact same tomato, same milk,

(a) National Council on Radiation Protection and Measurements (NCRP) and International Commission on Radiological Protection (ICRP). 


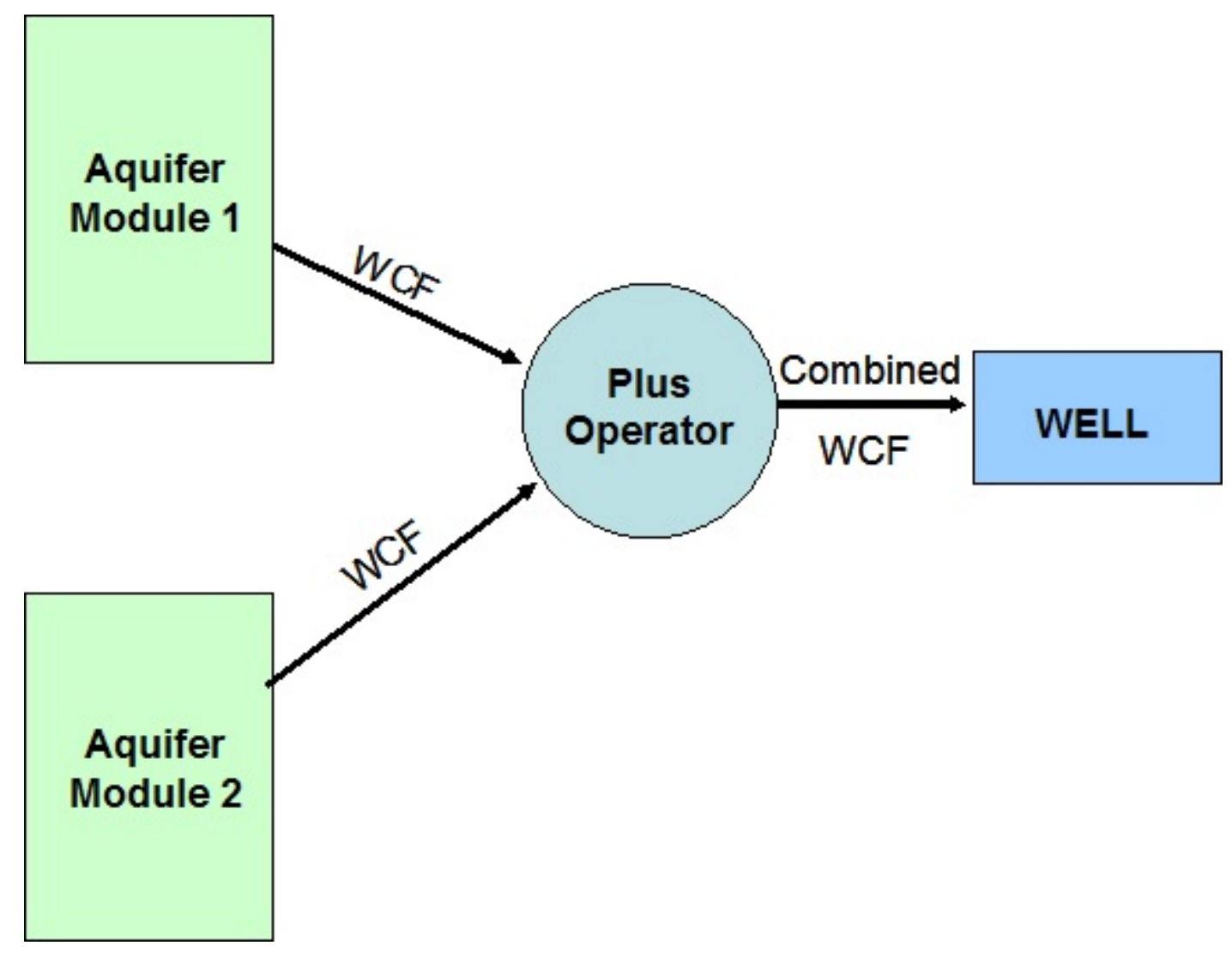

Figure 1.1. Application of the Plus Operator to Two Aquifer Plumes Contaminating the Same Well Location

same water, same soil). Therefore, the same person or population must be consuming the same foodstuff contaminated through two different sources.

5. Receptor Intake Files (RIFs): A limited set of superposition cases is possible for RIFs. Superposition is viable for the same person or population, the same exposure pathway (i.e., inhalation, ingestion, dermal contact, or external dose), the same foodstuff type (e.g., tomato, milk, meat, water, soil), the same chemical, the same times, and the same units. Different foodstuff media cannot be combined (e.g., tomatoes and water). The same person or population consumes the same foodstuff type, originating from different locations.

Of the five designs, only two (WFF and WCF) were implemented because three of the designs (ATO, EPF, and RIF) were more difficult to develop. The ATO, EPF, and RIF designs are described in this document, but the coding and testing were not implemented at this time.

This document provides draft requirements, design, data-file specifications, test plan, and the quality assurance/quality control (QA/QC) protocol for the Plus Operator. The requirements identify the attributes of the software. The design describes how the software will be structured to meet those requirements. The specification presents the specific modifications to FRAMES to meet the requirements and design. The test plan confirms that the basic functionality listed in the requirements (black box testing) actually functions as designed for the waterborne routes, and QA/QC confirms that the software meets the client's needs. 


\subsection{Requirements}

There are two sets of requirements necessary to fully define the operation of the Plus Operator within FRAMES. One set of requirements is for the FUI, and the other set is for the Plus Operator itself. Both sets of requirements are addressed below.

\subsection{Framework User Interface Requirements for Inclusion of the Plus Operator}

The FUI requirements for adding the Plus Operator into FRAMES are listed below. These are in addition to FUI requirements defined previously for FRAMES(Buck et al. 1999). Only new or modified FUI requirements are addressed below. The FUI must be capable of doing the following:

1. Determine if an implicit Plus Operator is required for the Conceptual Model developed.

2. Look "back" and "ahead" between module and data connections to determine if and what kind of Plus Operator is required to link modules and data sets.

3. Add a new "virtual" module on the input side of the Plus Operator (i.e., virtual-input module) to communicate to the upstream module (i.e., supplying information to be combined) the form of its output results. For example, if the downstream (i.e., receiving module) is another transporting medium, then the output results from the upstream module would be time-varying water and contaminant flux rates. If the connection is to an exposure module (e.g., at a well location), then the output would be time-varying concentrations. A "virtual" module is one defined by the FUI based on the Conceptual Model developed by the user.

4. Add a new "virtual" module on the output side of the Plus Operator (i.e., virtual-output module) to produce a new module that has the attributes of the modules whose results are being combined. For example, combining the data sets of two groundwater modules at a well should result in a combined data set that is consistent with information from one groundwater module at a well.

5. Include new parameters, defined by the FUI, for the virtual-input and -output modules and virtual-inputand -output-module types.

6. Allow the user to remove the Plus Operator created by the FUI from the Conceptual Model (drag to trash can).

\subsection{Plus Operator Requirements for Inclusion in FRAMES}

The Plus Operator is required to have certain attributes when it is included in the FRAMES' structure. At a minimum, the Plus Operator must be capable of doing the following:

1. Correctly combine results in a consistent format, both spatially and within the correct time steps, between two or more modules of the same FRAMES data file type:

- ATO: Atmospheric Transport Output file, containing time- and spatially-varying concentrations, deposition rates, and external dose (for radionuclides) for chronic models

- EPF: Exposure Pathway File, containing concentrations for each exposure pathway

- RIF: Receptor Intake File, containing dose rates for each exposure pathway 
- WCF: Water Concentration File, containing time-varying contaminant concentrations

- WFF: Water Flux File, containing time-varying water and contaminant fluxes.

2. Produce a single output file in the correct FRAMES data file format for a consuming (i.e., receiving) module

3. Be identifiable on the FUI interface to the user (added to the Conceptual Model by the FUI)

4. Interact with all other components of FRAMES, including the FUI and the appropriate modules

5. Combine a minimum of 2 and a maximum of 10 similar FRAMES data files into a single output file

6. Provide a user interface when necessary to allow the user to define the weighting factors and time steps for combining data. These user interfaces are critical to confirm that different data are combined appropriately for the receiving module.

\subsection{Plus Operator Input Requirements}

Other than providing a user interface when necessary to allow the user to define the weighting factors and time steps for combining data, no additional input requirements are anticipated, as the FUI would automatically modify the Global Input Data (GID) file to account for implementing the Plus Operator.

\subsection{Plus Operator Scientific Requirements}

The basic scientific requirement for the Plus Operator is that the results from two like data-file types (e.g., ATO, WFF, WCF) are additive, as described:

1. Water Flux Files: WFFs produce water flow rates (e.g., in $\mathrm{m}^{3} / \mathrm{yr}$ ) and contaminant mass flux rates (e.g., in $\left[\mathrm{g}\right.$ of contaminant] $/ \mathrm{yr}$ ), even if one is not used. The area (e.g., in $\left.\mathrm{m}^{2}\right)$ is also required.

a. Overland and Surface Water Module Types Producing Water Flux Files: Superposition on water flow rate $(\mathrm{Q})$ and contaminant mass flux rate $(\mathrm{Qc})$ is used primarily for overland and surface-water module types. The following information is passed between modules: area (A), water flow rate $(\mathrm{Q})$, and contaminant mass flux rate $(\mathrm{Qc})$. Each is calculated as follows:

i. Area: For FRAMES 1.x, the areas (e.g., in $\mathrm{m}^{2}$ ) are combined through superposition:

$A_{T}=A_{1}+A_{2}$. Superposition is assumed because the spacial location of the areas is unknown. For FRAMES 2.0, to combine areas for overland or surface-water module types, the areas have to touch or overlap, as illustrated in Figure 2.1. If the areas touch, then the areas are superimposed, and their vertices can be recomputed. If the areas overlap, then a combination of the areas is less than the sum of the areas, but the vertices can still be computed, as illustrated in Figure 2.1.

ii. Contaminant Mass Flux Rate: The contaminant mass flux rates (e.g., in [g of contaminant]/yr) can be superimposed: $\mathrm{Qc}_{\mathrm{T}}=\mathrm{Qc}_{1}+\mathrm{Qc}_{2}$. 


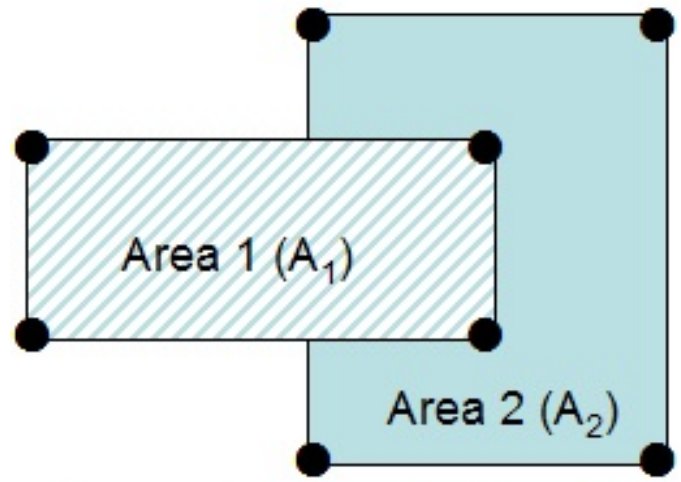

Areas prior to Plus Operator

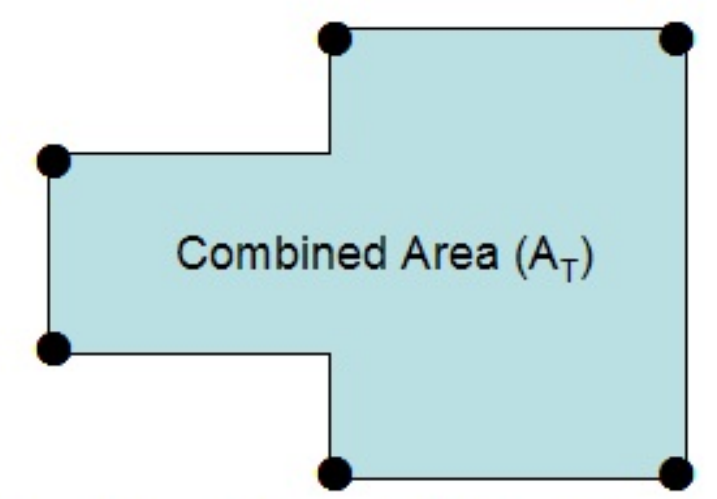

Combined Areas with Plus Operator

Figure 2.1. Approach to Account for Overlapping Areas when Combining like Overland or Surface-Water Modules

iii. Water Flow Rate: The water flow rates (e.g., in $\mathrm{m}^{3} / \mathrm{yr}$ ) can be superimposed: $\mathrm{Q}_{\mathrm{T}}=\mathrm{Q}_{1}+\mathrm{Q}_{2}$.

b. Vadose Zone and Aquifer Module Types Producing Water Flux Files: Superposition on water flow rate $(\mathrm{Q})$ and contaminant mass flux rate $(\mathrm{Qc})$ are used primarily for vadose zone and aquifer module types. The following information is passed between modules: area (A), water flow rate $(\mathrm{Q})$, and contaminant mass flux rate $(\mathrm{Qc})$. Each is calculated as follows:

i. $\quad$ Area: For FRAMES 1.x, the areas (e.g., in $\mathrm{m}^{2}$ ) have to be equivalent, although a representative control volume is associated with the representative concentration. The Plus Operator has to check and reconfirm that the areas are equivalent; therefore, $A_{1}=A_{2}=\ldots$. Equivalent areas will allow for an easier transition to FRAMES 2.0.

ii. Water Flow Rate: The water flow rates (e.g., in $\mathrm{m}^{3} / \mathrm{yr}$ ) can be superimposed: $\mathrm{Q}_{\mathrm{T}}=\mathrm{Q}_{1}+\mathrm{Q}_{2}$.

iii. Contaminant Mass Flux Rate: The contaminant mass flux rates (e.g., in [g of contaminant $] / \mathrm{yr}$ ) can be superimposed: $\mathrm{Q}_{\mathrm{cT}}=\mathrm{Q}_{\mathrm{c} 1}+\mathrm{Q}_{\mathrm{c} 2}$.

2. Water Concentration Files: WCFs produce contaminant concentrations (e.g., in [g of contaminant $] /\left(\mathrm{m}^{3}\right.$ of water]) at a given location. In FRAMES 1.x, a location is required, but in FRAMES 2.0, an area may be required with the concentration. Because superposition of concentrations assumes the same control volume, superposition of concentrations computes the new output from the Plus Operator. Using superposition of contaminant concentrations (e.g., in [g of contaminant $] /\left[\mathrm{m}^{3}\right.$ of water $]$ ):

$$
\mathrm{C}_{\mathrm{T}}=\mathrm{C}_{1}+\mathrm{C}_{2}
$$

If area is required, it has to be the same area: $\mathrm{A}_{1}=\mathrm{A}_{2}=\ldots .$. 
3. Air Transport Output Files: The ATO files produce deposition rates (D) (e.g., in [kg of contaminant $] / \mathrm{m}^{2} / \mathrm{yr}$ ) and air concentrations (C) (e.g., in [ $\mathrm{kg}$ of contaminant $] /\left[\mathrm{m}^{3}\right.$ of air $]$ ) at a given location for the chronic models. For deposition rates, this result is associated with an area. To combine deposition rates, superposition can be used, but the deposition rates have to be associated with the same area (i.e., $\left.\mathrm{A}_{1}=\mathrm{A}_{2}=\ldots ..\right)$ :

$$
\mathrm{D}_{\mathrm{T}}=\mathrm{D}_{1}+\mathrm{D}_{2}
$$

Air concentrations are annual concentrations and can likewise be combined using superposition, but it must be associated with the same receptor location,

$$
\mathrm{C}_{\mathrm{T}}=\mathrm{C}_{1}+\mathrm{C}_{2}
$$

This approach will not work for acute air concentrations, so one can only obtain acute air concentrations by computing on an hourly basis.

4. Exposure Pathway Files: EPFs produce environmental concentrations (i.e., in [mg contaminant] $/[\mathrm{kg}$ of wet matrix], [mg contaminant]/[L water], or [mg contaminant $] /\left[\mathrm{m}^{3}\right.$ air $]$ ) in foodstuff before consumption. A limited set of superposition cases is possible for EPFs. Superposition is viable for the same foodstuff medium (e.g., tomatoes, water, air, or soil), the same chemical, the same times, the same location, and the same concentration units. When referring to the same foodstuff medium, superposition is implemented on the exact same foodstuff (e.g., exact same tomato, same milk, same water, same soil). Therefore, the same person or population must be consuming the same foodstuff that is contaminated through two different sources:

$$
\mathrm{C}_{\mathrm{T}}=\mathrm{C}_{1}+\mathrm{C}_{2}
$$

5. Receptor Intake Files: RIFs produce daily intake rates for ingestion (i.e., in $\mathrm{mg} / \mathrm{kg} / \mathrm{d}$ ) and inhalation (i.e., in $\mathrm{mg} / \mathrm{kg} / \mathrm{d}$ or $\mathrm{mg} / \mathrm{m}^{3}$ ). A limited set of superposition cases is possible for RIFs. Superposition is viable for the same person or population, the same exposure pathway (i.e., inhalation, ingestion, dermal contact, or external dose), the same foodstuff type (e.g., tomato, milk, meat, water, soil), the same chemical, the same times, and the same units. Different foodstuff media cannot be combined (e.g., tomatoes and water). The same person or population consumes the same foodstuff type, originating from different locations (i.e., source of foodstuff, not location of the original source of contamination):

a. For combining ingestion rates $\left(\mathrm{Ig}_{\mathrm{R}}\right)$ :

$$
\operatorname{Ig}_{\mathrm{RT}}=\operatorname{Ig}_{\mathrm{R} 1}+\operatorname{Ig}_{\mathrm{R} 2}
$$

b. For combining inhalation rates $\left(\operatorname{In}_{\mathrm{R}}\right)$ :

$$
\mathrm{In}_{\mathrm{RT}}=\mathrm{In}_{\mathrm{R} 1}+\mathrm{In}_{\mathrm{R} 2}
$$

Superposition on EPFs assumes that the same foodstuff (e.g., the same exact tomato) is contaminated from two different sources, and superposition on RIFs assumes that the same type of foodstuff (e.g., category of tomato, that is, two different tomatoes, not the same tomato) is combined from two different locations. 


\subsection{Plus Operator Output Requirements}

The output associated with the Plus Operator must have the same format as that associated with the input data file type. 


\subsection{Design}

This section describes the initial design of the Plus Operator module in FRAMES. The Plus Operator can add a number of WFFs, WCFs, ATO files, EPFs, or RIFs. When implementing a Plus Operator, the connection protocol between the two modules becomes critical. A relationship exits between an upstream module, which produces information, and a downstream module, which consumes information. For example, in Figure 3.1, the aquifer modules AQ1 and AQ2 represent upstream modules, whose results are eventually consumed by the downstream exposure module (EXP1).

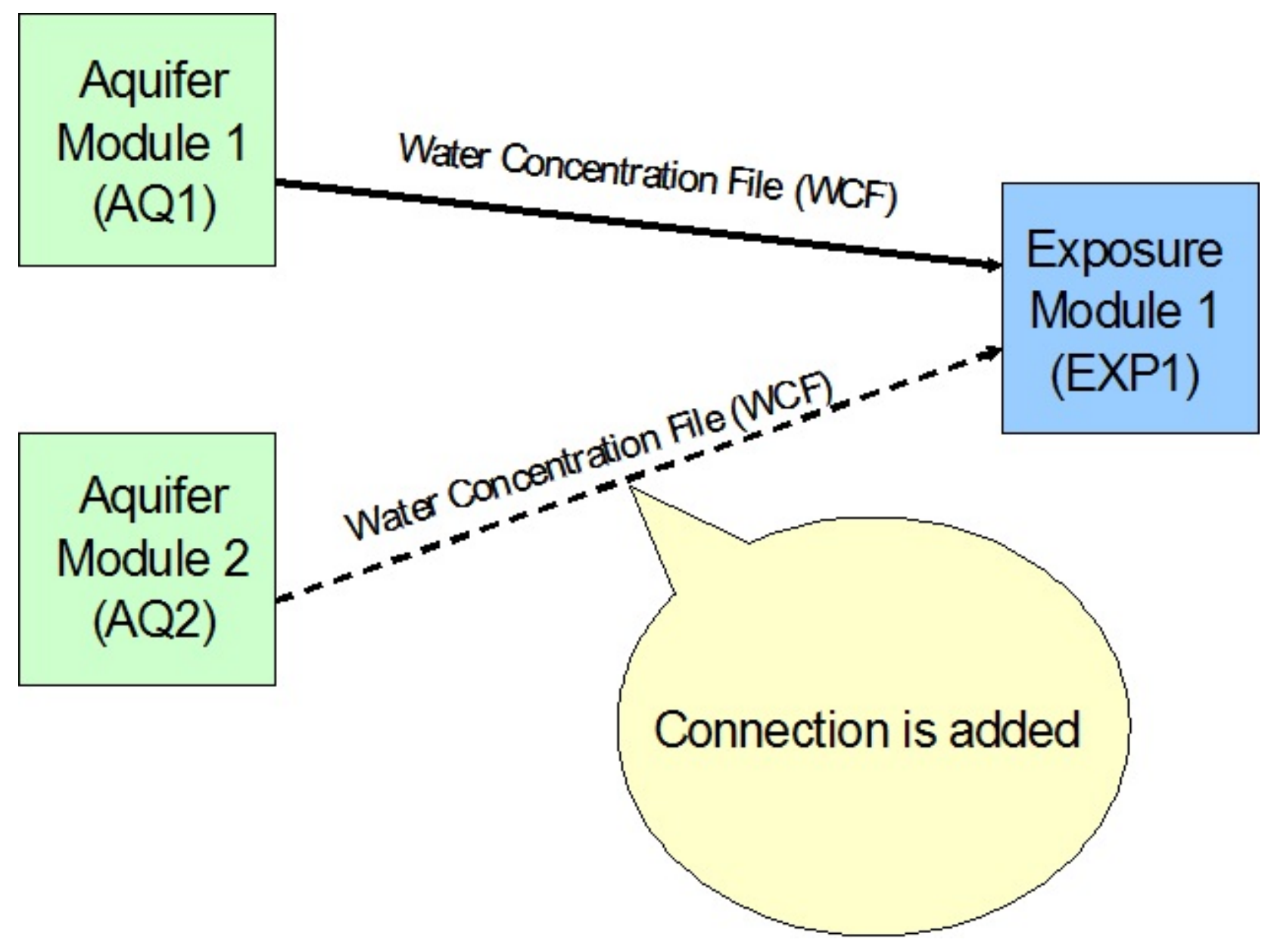

Figure 3.1. Conceptual Model Developed by User in FRAMES

The output results associated with the upstream module can be influenced by the type of downstream module. For example, an aquifer module would look "forward" (i.e., downstream) to see if the connection is to a transport or an exposure module. If the connection is to another transport module, then the aquifer module would produce a WFF. If the connection is to an exposure module, then a WCF is produced. When attempting to use the Plus Operator, the issue of "transparency" reveals itself. Figure 3.2 illustrates the use of a Plus Operator when combining two aquifers at an exposure point. AQ1 and AQ2 represent contaminant plumes in two different aquifers that impact the same exposure-point location, providing time-varying 
concentrations for use in the exposure module (i.e., EXP1). In other words, water is withdrawn from two different wells and is used simultaneously at the same exposure location. As Figure 3.2 reveals, when the aquifer modules look forward to the downstream module, they only see the module representing the Plus Operator, in effect, masking the connectivity between the aquifers and the point of exposure. The aquifer modules would not know whether to produce a WFF or WCF.

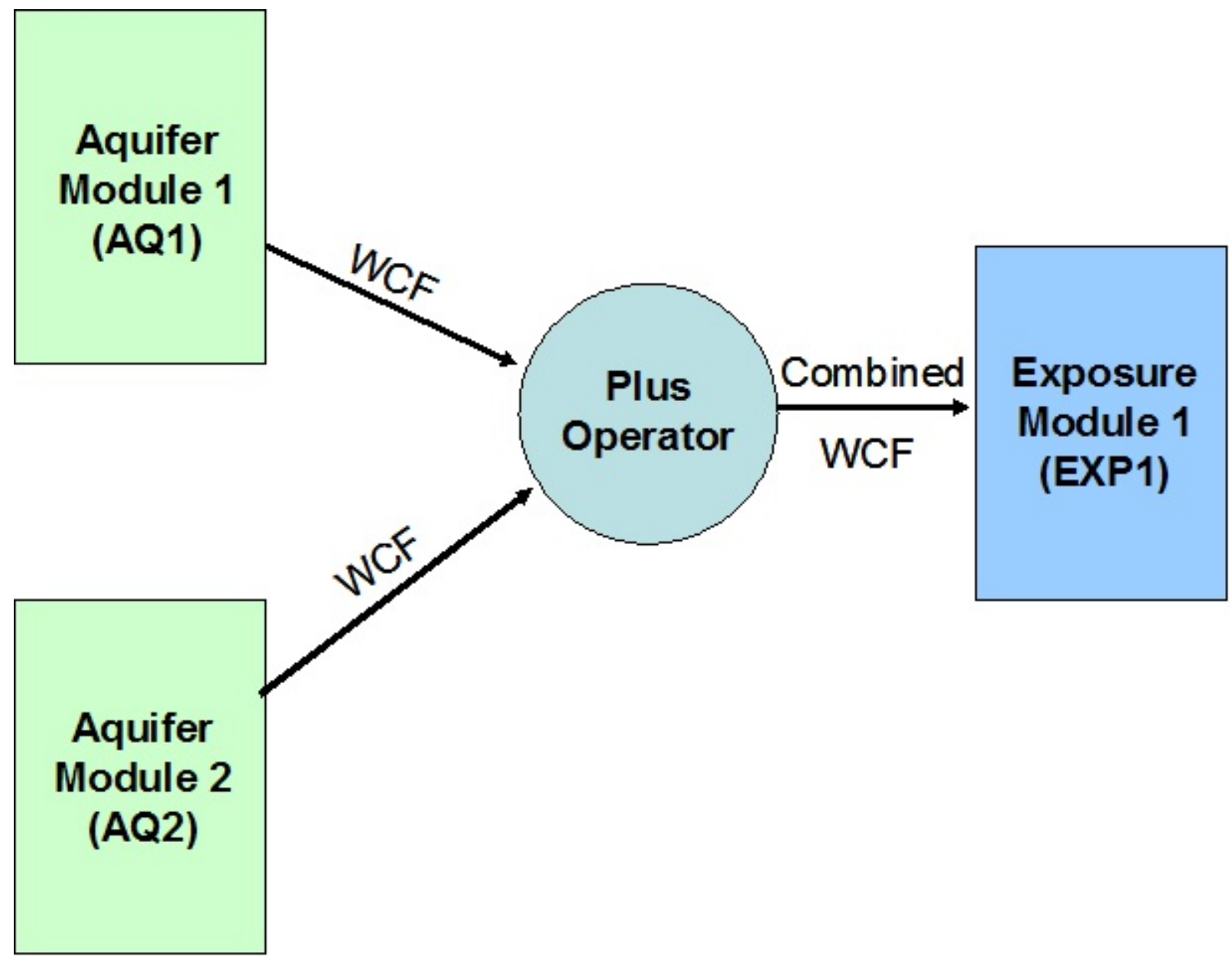

Figure 3.2. Plus Operator Combining WCF Results from Two Different Aquifer Modules to a Receiving Exposure

Conversely, the downstream module "looks back" and "sees" which medium type produces the data to be consumed. For example, the exposure module typically looks back to see if an aquifer or a surface-water module produces the water concentrations that it consumes at the exposure point. Looking back is needed to confirm that the user is not asked whether an exposure-point concentration should be calculated for inappropriate combinations of module results (e.g., boating in the aquifer). If a module is receiving information from an upstream module, it expects to see a certain data-type file. For example, if the exposure module is receiving aquifer concentrations, it expects to see an upstream aquifer module with a format matching that of a WCF. When attempting to use the Plus Operator, the issue of "transparency" reveals itself again. As Figure 3.2 reveals, when the exposure module looks back at the upstream module, it only sees the 
module representing the Plus Operator. The exposure module would not be capable of distinguishing between surface water or aquifer results (i.e., transparency problem) because the Plus Operator, not the upstream module, is directly connected.

A design to solve this problem is an implicit invocation of the Plus Operator. That is, the user would never explicitly add the Plus Operator. If the Plus Operator were implied by the restrictions of the exposure module, then the FUI would invoke the Plus Operator, as needed. Figure 3.1 presents the example of a user attempting to add two aquifer (i.e., AQ1 and AQ2) results together at one exposure location (EXP1). Unfortunately, the example exposure module has the restriction that it can accept only one aquifer connection (i.e., only one WCF). Without the Plus Operator, the current FUI would not allow Figure 3.3 to be drawn. But if the FUI were changed (i.e., a module can accept an input of a given type [e.g., WCF, WFF]), the FUI would let the user continue, and the FUI would store information describing the invocation of the Plus Operator. The Conceptual Model could even be modified to show this assumption as displayed in Figure 3.3.

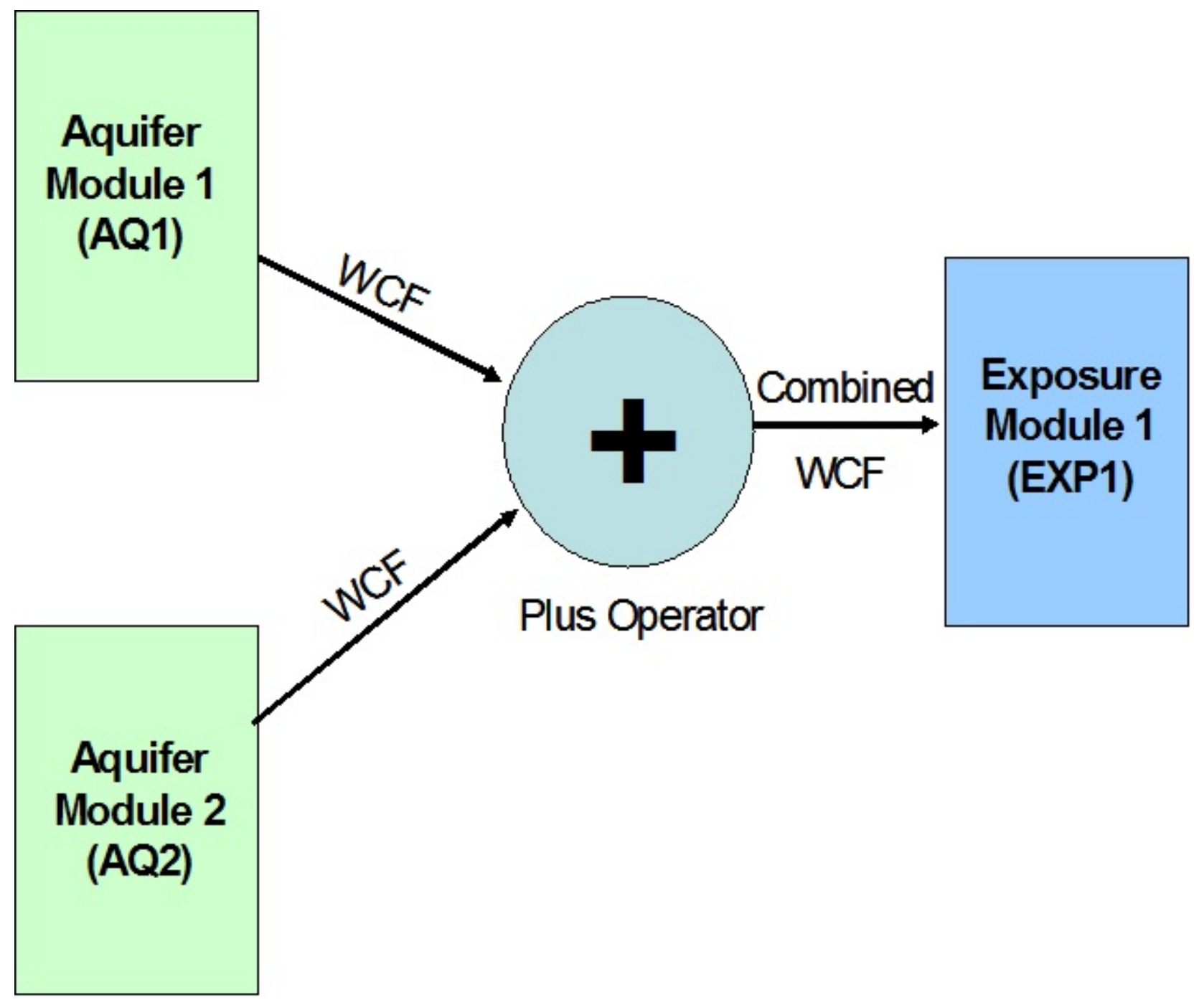

Figure 3.3. Conceptual Model With Plus Operator Added by the FUI, Based on the User's Original Problem 
To invoke the Plus Operator, as described in Figure 3.3, yet maintain the capability of modules to look forward and backward, as appropriate, the FUI develops a Plus Operator that automatically accounts for and contains forward- and backward-looking attributes. Figure 3.4 shows a virtual split of the Plus Operator into two separate modules. In effect, the Plus Operator subdivides into a virtual module of the same type as the original consuming (i.e., receiving downstream) module, and a virtual module of the same type of the original producing (i.e., upstream) modules that are being combined. Figure 3.4 presents an example of the "new" virtual Plus Operator, which represents an expansion of Figure 3.3. In this illustrative figure, two aquifer modules are being combined at a single exposure location. The Plus Operator automatically inspects the connections and establishes virtual modules to allow for proper viewing, either forward looking from the upstream modules (AQU1 and AQU2) or backward looking from the downstream module (EXP1). Because the aquifer modules expect to see an exposure module, a virtual exposure module (EXP16) is created. Likewise, because the downstream exposure module (EXP1) expects to see an aquifer module, a virtual aquifer module is created (AQU16). The FUI only needs to keep track of EXP16 and AQU16. To account for these two new virtual modules, three new parameters are added to the FUI section of the GID file, which is the file that contains all of the input parameters for the modules describing a given scenario. The three new parameters are 1) NumVirt(Scenario) for the number of virtual connections in the current scenario, 2) VirtFirst(Scenario,NumVirt), which is the first module's name and is always the first module in the execution sequence, and 3) VirtSecond(Scenario,NumVirt), which is the second module's name. For Figure 3.4, NumVirt=1, VirtFirst=EXP16, and VirtSecond=AQU16.

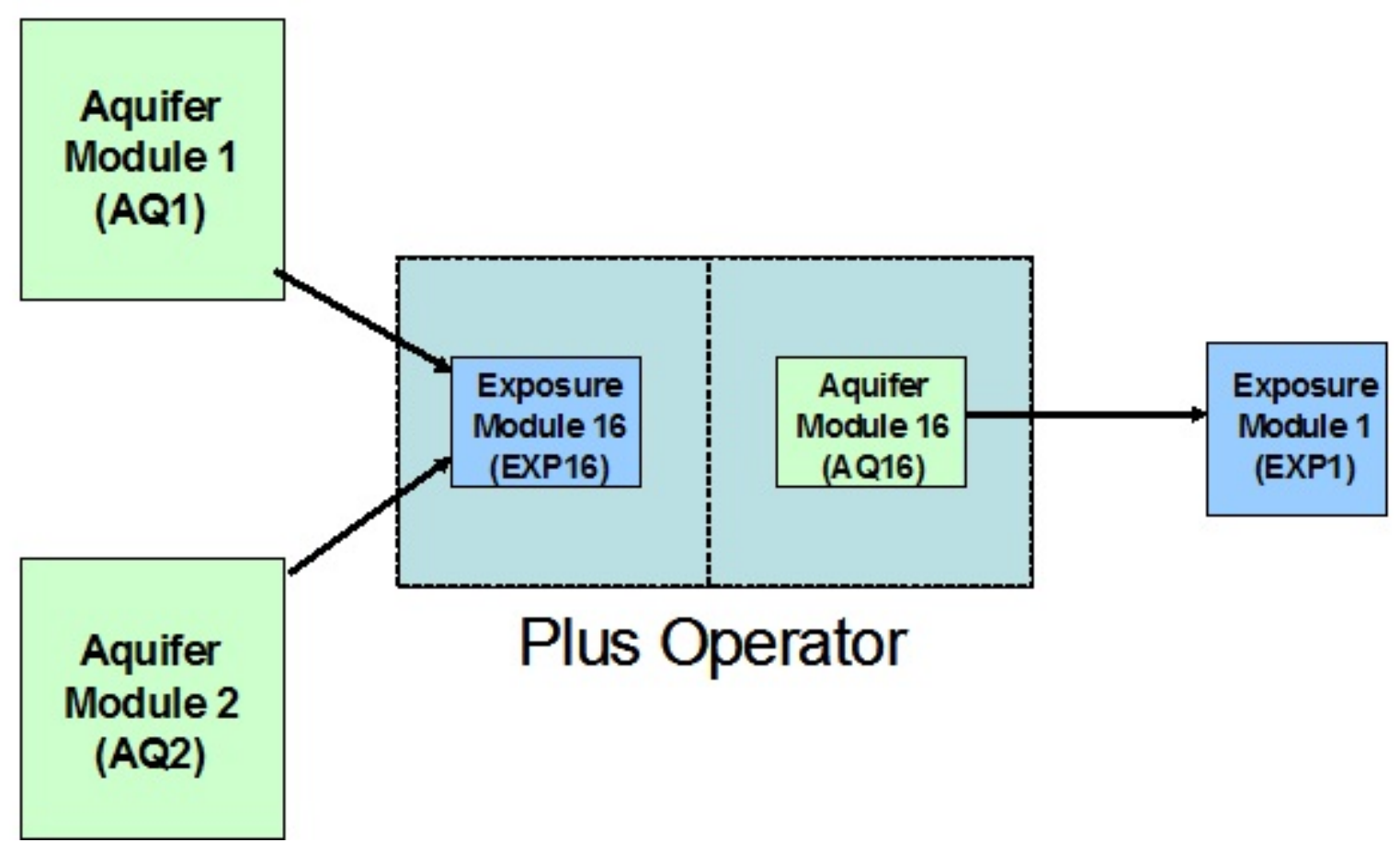

Figure 3.4. Virtual Modules Created by the FUI to Implement the Plus Operator for the Existing Conceptual Model 
Each time a module is simulated or the user draws the Conceptual Model, the FUI uses this information and checks to see if the module that had just executed has any virtual connections. If it does, the FUI changes the state of the VirtSecond to match the state of the VirtFirst module. This change allows the rest of the FUI logic for module execution to remain unchanged. During the update of the Conceptual Model, the FUI uses the VirtSecond parameter to know which module to "skip" during the drawing process. If the Plus Operator is deleted, both the EXP16 and AQU16 module information would need to be removed. The advantages of this approach are as follows.

1. The logic of the FUI is only changed as follows:
a. Recognize the need for the Plus Operator when the user is creating the Conceptual Model picture.
b. Add two modules (not one) when a Plus Operator is needed.
c. Skip drawing one of the module icons (may not be required because of overlap).
d. Synchronize the state and screen locations of virtually equivalent modules.
e. Remove virtually equivalent modules together if the user deletes the Plus Operator.

2. The logic of all the current modules in FRAMES does not need to be changed, resulting in a tremendous savings to FRAMES. The FUI takes on all the changes and leaves the module requirements unchanged. 


\subsection{Date File Specifications}

Before the Plus Operator is added by the FUI, the media data in the GID file would be as follows, assuming that the drawing is the first scenario in the FUI:

$\operatorname{AquNum}(1)=2$

AquName $(1,1)="$ Aqu5"

AquLabel $(1,1)="$ Aq1"

AquName $(1,2)="$ Aqu 8 "

AquLabel $(1,2)="$ Aq2"

$\operatorname{ExpNum}(1)=1$

ExpName $(1,1)=" \operatorname{Exp} 9 "$

ExpLabel $(1,1)="$ Exp"

ExpTypeNum $(1,1)=2$

ExpType $(1,1,1)="$ Aquifer"

ExpType $(1,1,2)="$ Aquifer"

ExpSrcName $(1,1,1)="$ Aqu5"

ExpSrcName $(1,1,2)=" A q u 8 "$

The FUI would change the connections between modules by:

1. Adding a new medium that matches the type of medium on the input side of the Plus Operator (an aquifer module in the example).

2. Adding a new medium that matches the type of medium on the output side of the Plus Operator (an exposure module in the example).

3. Taking the references from the exposure module back to the aquifer modules and moving them to the new exposure module just defined.

4. Having the exposure module now refer to the just-added aquifer module.

Assuming that the virtual aquifer and exposure modules are assigned the names AQU16 and EXP16, respectively, the new media data would be as follows:

$\operatorname{AquNum}(1)=3$

AquName $(1,1)="$ Aqu5"

AquLabel $(1,1)="$ Aq1"

AquName $(1,2)="$ Aqu $8 "$

AquLabel $(1,2)="$ Aq2"

AquName $(1,3)="$ Aqu16"

AquLabel $(1,3)=$ "Plus"

$\operatorname{AquSrcNum}(1,3)=1$

AquSrcName $(1,3,1)=" E x p 16 "$

$\operatorname{ExpNum}(1)=2$

ExpName $(1,1)=" E x p 9 "$

ExpLabel $(1,1)=" E x p "$

ExpTypeNum $(1,1)=1$

ExpType $(1,1,1)="$ Aquifer"

ExpSrcName $(1,1,1)="$ Aqu 16"

ExpName $(1,2)=" E x p 16 "$ 
ExpLabel $(1,2)=" P l u s "$

ExpTypeNum $(1,2)=2$

ExpType $(1,2,1)="$ Aquifer"

ExpSrcName $(1,2,1)="$ Aqu5"

ExpType $(1,2,2)="$ Aquifer"

$\operatorname{ExpSrcName}(1,2,2)="$ Aqu $8 "$

As noted earlier, the GID file would contain NumVirt(Scenario), VirtFirst(Scenario,NumVirt), and VirtSecond(Scenario,NumVirt):

NumVirt $=1$

VirtFirst="Exp16"

VirtSecond="Aqu16" 


\subsection{Test Plan and Results}

The following test cases were designed to test and confirm that the Plus Operator meets all of its requirements. The Plus Operator was initially coded for only the water-based modules. Therefore, the following test cases only address adding WFF and WCF file types. Coding for the ATO, EPF, and RIF Plus Operators has not been performed yet. Two contaminants will be used in the test cases: benzene and strontium-90, the latter having one decay product (yttrium-90). Hand calculations for each of the test cases have been developed and are included in the file "Plus Operator Hand Calcs02.xls."

\subsection{Test Case PlusOp01}

This test case evaluates the capability of the Plus Operator to correctly add the WFF output from two river modules. The time steps for each river module output are identical, requiring the Plus Operator to simply add results. The input data can be found in the file PlusOp01.gid. It is expected that the Plus Operator would add the results and produce information to populate the output file PlusOp01.wff.

When the test was executed, the Plus Operator passed. The results were checked against the hand calculations.

\subsection{Test Case PlusOp02}

This test case evaluates the capability of the Plus Operator to correctly add the WFF output from two vadose zone modules. The time steps for each vadose zone module output are identical, requiring the Plus Operator to simply add results. The input data can be found in file PlusOp02.gid. It is expected that the Plus Operator would add the results and produce information to populate the output file PlusOp02.wff.

When the test was executed, the Plus Operator passed. The results were checked against the hand calculations.

\subsection{Test Case PlusOp03}

This test case evaluates the capability of the Plus Operator to correctly add the WFF output from two aquifer modules. The time steps for each aquifer module output are identical, requiring the Plus Operator to simply add results. The input data can be found in the file PlusOp03.gid. It is expected that the Plus Operator would add the results and produce information to populate the output file PlusOp03.wff.

When the test was executed, the Plus Operator passed.. The results were checked against the hand calculations.

\subsection{Test Case PlusOp04}

This test case evaluates the capability of the Plus Operator to correctly add the WCF output from two river modules. The time steps for each river module output are identical, requiring the Plus Operator to simply add results. The input data can be found in the file PlusOp04.gid. It is expected that the Plus Operator would add the results and produce information to populate the output file PlusOp04.wcf. 
When the test was executed, the Plus Operator passed. The results were checked against the hand calculations.

\subsection{Test Case PlusOp05}

This test case evaluates the capability of the Plus Operator to correctly add the WCF output from two aquifer modules. The time steps for each aquifer module output are identical, requiring the Plus Operator to simply add results. The input data can be found in the file PlusOp05.gid. It is expected that the Plus Operator would add the results and produce information to populate the output file PlusOp05.wcf.

When the test was executed, the Plus Operator passed. The results were checked against the hand calculations.

\subsection{Test Case PlusOp06}

This test case evaluates the capability of the Plus Operator to correctly add the WCF output from two aquifer modules. The time steps for each aquifer module output are different, requiring the Plus Operator to interpolate points in each time series before adding results. The input data can be found in the file PlusOp06.gid. It is expected that the Plus Operator would add the results and produce information to populate the output file PlusOp06.wcf.

When the test was executed, the Plus Operator passed. The results were checked against the hand calculations.

\subsection{Test Case PlusOp07}

This test case evaluates the capability of the Plus Operator to correctly add the WFF output from more than two river modules. Three river modules provide output to be added together. The time steps for each river module output are identical, requiring the Plus Operator to simply add results. The input data can be found in the file PlusOp07.gid. It is expected that the Plus Operator would add the results and produce information to populate the output file PlusOp07.wff.

When the test was executed, the Plus Operator passed. The results were checked against the hand calculations. 


\subsection{Quality Assurance Program}

The Plus Operator was developed under a quality assurance program documented in Gelston et al. (1998). Quality is defined as the capability of the software to meet client needs. Meeting client needs starts with a shared understanding of how the software must perform and continues throughout the software life cycle of design, development, testing, and implementation through attention to details.

Figure 6.1 outlines the software-development process that was used for the Plus Operator, highlighting the quality check points. The Plus Operator activities flow down the left side of Figure 6.1 because it is software developed for the first time as opposed to a modification to existing software. The process shown is designed for compatibility with similar processes used by other government agencies. For example, this quality process compares favorably with that in U.S. Environmental Protection Agency (EPA) Directive 2182, "System Design and Development Guidance" (EPA 1997). It also compares favorably with the Office of Civilian Radioactive Waste Management's Quality Assurance Requirements and Description, Supplement I, Software (OCRWM 1995). Activities roughly equivalent across these processes are shown in Table 6.1.

Development of the Plus Operator includes implementing a quality assurance checklist (see Figure 6.2). All team members understand the component requirements and design necessary to confirm quality. Completion of this checklist verifies that all documentation will be complete for transfer of the software to client use. 


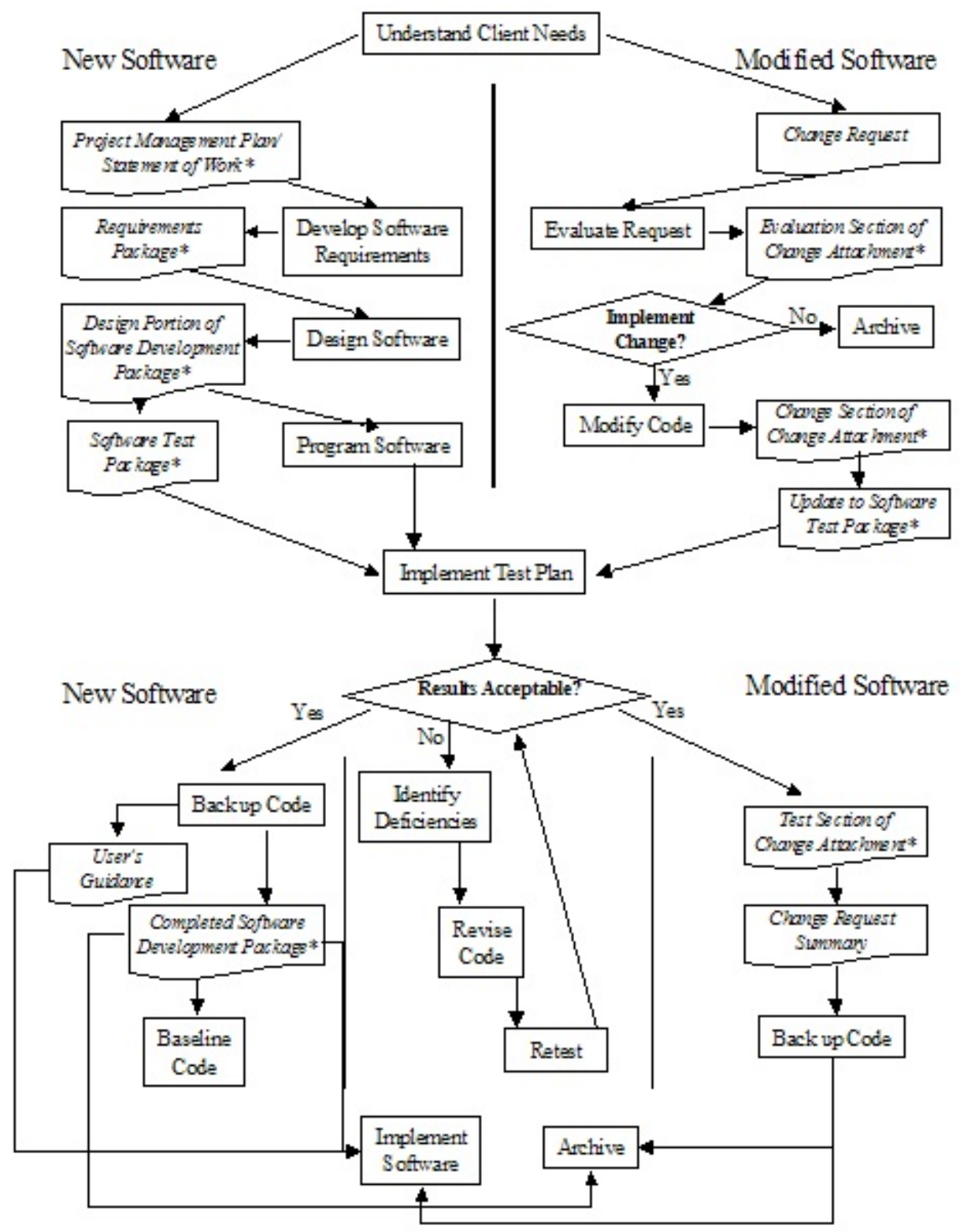

Figure 6.1. Confirming Quality in the Environmental Software Development Process (* indicates quality review stage; box with wavy bottom line and italics font indicate a document rather than an activity) 
Table 6.1. Relationship of PNNL Environmental Software Development Process to Quality Assurance Requirements

\begin{tabular}{|c|c|c|}
\hline $\begin{array}{l}\text { OCRWM Quality Assurance } \\
\text { Requirement }^{\text {(a) }}\end{array}$ & \begin{tabular}{|l|} 
EPA Essential Element of \\
Information $^{(b)}$
\end{tabular} & $\begin{array}{l}\text { Environmental Software } \\
\text { Process Equivalent (Section) }\end{array}$ \\
\hline & $\begin{array}{l}\text { 4-System Implementation } \\
\text { Plan }\end{array}$ & $\begin{array}{l}\text { Project Management Plan or } \\
\text { Statement of Work }\end{array}$ \\
\hline $\begin{array}{l}\text { I.2.5A Functional Requirements } \\
\text { Information Documentation; I.2.5C } \\
\text { Requirements and Design } \\
\text { Documentation }\end{array}$ & $\begin{array}{l}\text { 5-System Detailed } \\
\text { Requirements Document }\end{array}$ & Requirements Package \\
\hline $\begin{array}{l}\text { I.2.1 Software Life Cycles, Baselines } \\
\text { (see their Appendix C), and Controls }\end{array}$ & $\begin{array}{l}\text { 6-Software Management } \\
\text { Plan }\end{array}$ & $\begin{array}{l}\text { Project Management Plan or } \\
\text { Statement of Work and Gelston } \\
\text { et al. (1998) }\end{array}$ \\
\hline $\begin{array}{l}\text { I.2.2 Software Verification }{ }^{(\mathrm{c})} \text { and } \\
\text { Software Validation; } \\
\text { I.2.4 Software Validation }^{(\mathrm{d})}\end{array}$ & $\begin{array}{l}\text { 7-Software Test and } \\
\text { Acceptance Plan }\end{array}$ & Software Test Package \\
\hline $\begin{array}{l}\text { I.2.3 Software Verification; } \\
\text { I.2.5C Requirements and Design } \\
\text { Information Documentation }\end{array}$ & $\begin{array}{l}\text { 8-Software Design } \\
\text { Document }\end{array}$ & $\begin{array}{l}\text { Design Portion of Software } \\
\text { Development Package }\end{array}$ \\
\hline I.2.6A Configuration Identification & & $\begin{array}{l}\text { Completed Software } \\
\text { Development Package }\end{array}$ \\
\hline \multirow{2}{*}{$\begin{array}{l}\text { I.2.6B Configuration Control; I.2.6C } \\
\text { Configuration Status; I.2.7 Defect } \\
\text { Reporting and Resolution }^{(\mathrm{e})}\end{array}$} & $\begin{array}{l}\text { 9-Software Maintenance } \\
\text { Document }\end{array}$ & Modification Documentation \\
\hline & $\begin{array}{l}\text { 10-Software Operations } \\
\text { Document }\end{array}$ & User's Guidance and Training \\
\hline \multirow[t]{2}{*}{$\begin{array}{l}\text { I.2.5B User Information } \\
\text { Documentation }\end{array}$} & $\begin{array}{l}\text { 11-Software User's } \\
\text { Reference Guide }\end{array}$ & User's Guidance and Training \\
\hline & $\begin{array}{l}\text { 12-System Integration } \\
\text { Test Reports }\end{array}$ & Software Test Package \\
\hline
\end{tabular}

(a) Note that OCRWM requirement I.2.8, Control of the Use of Software, is the responsibility of the OCRWM-related client.

b) Elements 1 through 3 are generally completed by clients in the U.S. Environmental Protection Agency before contract initiation with the project team.

(c) Verification includes informal code testing by software engineers to confirm that the code functions as required.

(d) Validation includes testing by those other than the software engineers who developed the code to provide an independent confirmation that the software functions as required.

(e) Note that some changes requested by clients may not be made in the software unless funding has been allocated for such modifications. 
A. General Requirements Analysis

--Documented in Statement of Work

--Contains information on (all of the following) problem description deliverables project team capabilities to be used restrictions difficulties envisioned compatibilities with existing software/hardware scope of the project

B. Specific Requirements Analysis

--Documented in requirements section of documentation.

--Contains information on (all of the following) purpose of the software structure of the software hardware and software requirements input and output requirements scientific basis assumptions limitations

C. Design Documentation

--Documented in design portion of documentation. team task plans/Project Management Plan

--Contains information on (all of the following) code type and description development team members specifications logic diagrams "help" descriptions methods to confirm consistency in components mathematical formulations need for pre/post-processors

D. Development Documentation

--Documented in Specifications Document Quality Assurance Archive

--Contains information on (all of the following) baseline hard copy of the source code diskette copy name of computer language(s) used

E. Testing Documentation --Documented in

Figure 6.2. Quality Assurance Implementation Checklist for Plus Operator 


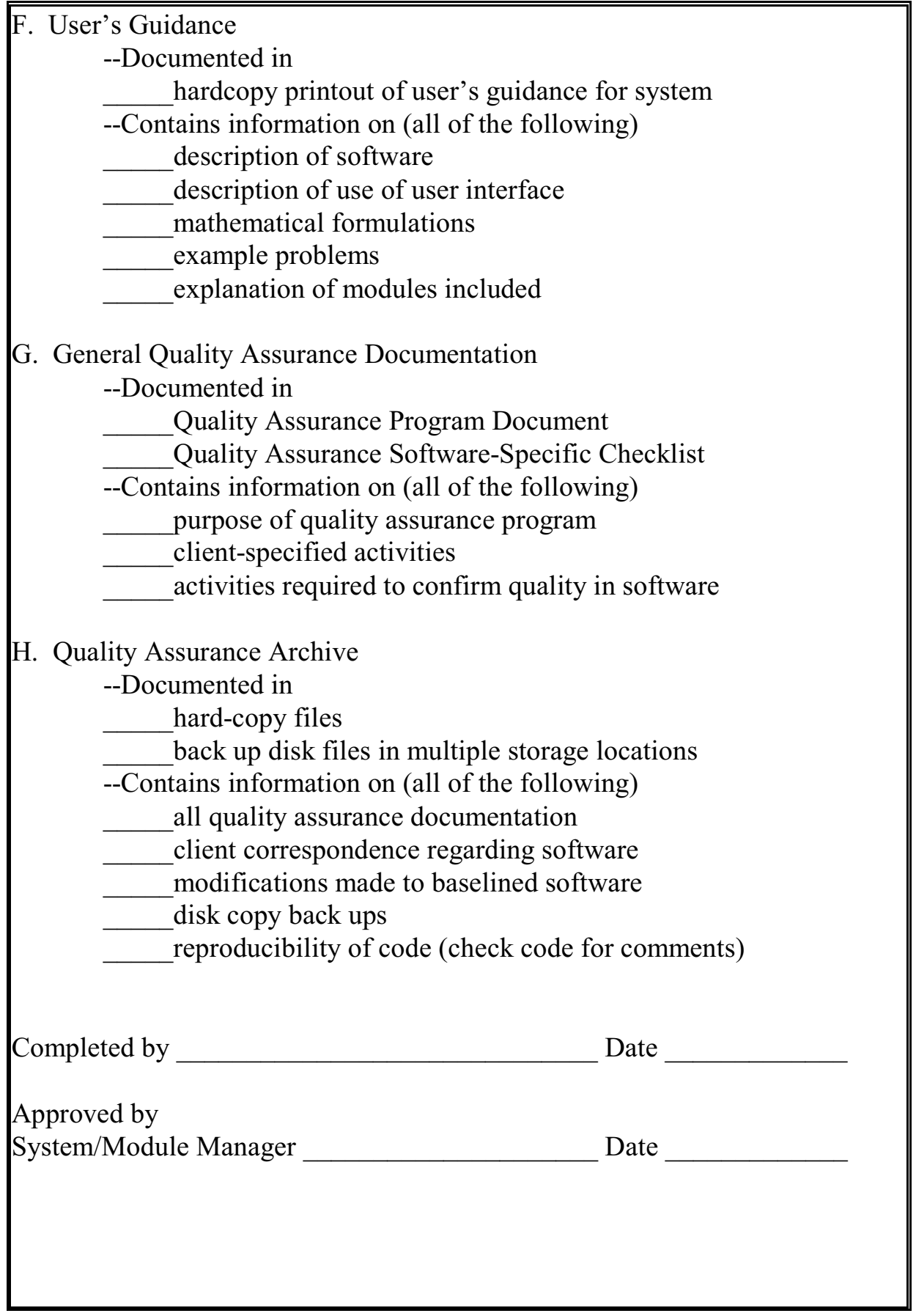

Figure 6.2. Quality Assurance Implementation Checklist for Plus Operator (contd) 


\subsection{References}

Buck JW, BL Hoopes, KJ Castleton, and RY Taira. 1999. Requirements for the FRAMES User Interface PNNL-SA-32277, Pacific Northwest National Laboratory, Richland, WA.

Gelston GM, RE Lundgren, JP McDonald, and BL Hoopes. 1998. An Approach to Ensuring Quality in Environmental Software. PNNL-11880, Pacific Northwest National Laboratory, Richland, WA.

Office of Civilian Radioactive Waste Management (OCRWM). 1995. Quality Assurance Requirements and Description, Supplement I, Software. Office of Civilian Radioactive Waste Management, U.S. Department of Energy, Washington, D.C.

U.S. Environmental Protection Agency (EPA). 1997. System Design and Development Guidance. EPA Directive Number 2182, U.S. Environmental Protection Agency, Washington, D.C. 